\title{
Potentially zoonotic gastrointestinal nematodes co-infecting free ranging non-human primates in Kenyan urban centres
}

\author{
Peris Mbuthia $^{1}$ (D) | Edwin Murungi ${ }^{2}$ | Vincent Owino ${ }^{1}$ | Mercy Akinyi ${ }^{3}$ | \\ Gillian Eastwood $^{4}$ | Richard Nyamota ${ }^{1}$ (D) | Isaac Lekolool ${ }^{5}$ | Maamun Jeneby ${ }^{6}$
}

${ }^{1}$ Department of Biochemistry and Molecular Biology, Egerton University, Egerton, Kenya

${ }^{2}$ Department of Medical Biochemistry, Kisii University, Kisii, Kenya

${ }^{3}$ Animal Sciences Department, Institute of Primate Research (IPR), Karen, Kenya

${ }^{4}$ Virginia Polytechnic Institute \& State University, College of Agriculture \& Life Sciences, Virginia Tech, Blacksburg, VA, USA

${ }^{5}$ Kenya Wildlife Service, Nairobi, Kenya

${ }^{6}$ Zoonoses Unit, Tropical Infectious Diseases Department, Institute of Primate Research (IPR), Karen, Kenya

Correspondence

Peris Mbuthia, Department of Biochemistry and Molecular Biology, Egerton University, P.O. Box 536- 20115 Egerton, Kenya. Email: mbuthiaperis8@gmail.com

Funding information

The Consortium for National Health Research (CNHR) grant, Kenya; Global Enhancement Fund grant, USA (2017)

\begin{abstract}
Background: Natural infections with soil-transmitted nematodes occur in non-human primates (NHPs) and have the potential to cross primate-species boundaries and cause diseases of significant public health concern. Despite the presence of NHPs in most urban centres in Kenya, comprehensive studies on their gastrointestinal parasites are scant.

Objective: Conduct a cross-sectional survey to identify zoonotic nematodes in freeranging NHPs found within four selected urban and peri-urban centres in Kenya.

Methods: A total of 86 NHPs: 41 African green monkeys [AGMs] (Chlorocebus aethiops), 30 olive baboons (Papio anubis), 5 blue monkeys (Cercopithecus mitis stuhlmanni) and 10 red-tailed monkeys (Cercopithecus ascanius) were sampled once in situ and released back to their habitat. Microscopy was used to identify nematodes egg and larvae stages in the samples. Subsequently, PCR coupled with high-resolution melting (PCR-HRM) analysis and sequencing were used to identify nodule worms.

Results: NHPs inhabiting densely populated urban environs in Kenya were found infected with a rich diversity of nematodes including three potentially zoonotic nematodes including Oesophagostomum stephanostomum, Oesophagostomum bifurcum and Trichostrongylus colubriformis and co-infections were common.

Conclusion: Phylogenetic analysis showed that $\mathrm{O}$. stephanostomum from red-tailed and blue monkeys have a close evolutionary relatedness to human isolates suggesting the zoonotic potential of this parasite. Moreover, we also report the first natural co-infection of $O$. bifurcum and $O$. stephanostomum in free-ranging AGMs.
\end{abstract}

\section{KEYWORDS}

non-human primates, Oesophagostomum, PCR-HRM, urban, zoonoses

\section{1 | INTRODUCTION}

Free-ranging non-human primates (NHPs) are a source of information regarding maintenance, transmission and disease dynamics of public health importance (Eastwood et al., 2017; Kooriyama et al., 2012; Kouassi et al., 2015). Of concern are soil-transmitted helminths (STHs) whose partial development outside their hosts allows persistence of infective stages in the environment, enabling transmission between

This is an open access article under the terms of the Creative Commons Attribution-NonCommercial-NoDerivs License, which permits use and distribution in any medium, provided the original work is properly cited, the use is non-commercial and no modifications or adaptations are made.

(c) 2021 The Authors. Veterinary Medicine and Science Published by John Wiley \& Sons Ltd 
closely related host species (Cibot et al., 2015; Ghai, Chapman, et al., 2014; Ghai, Simons, et al., 2014). Several studies have described cases of zoonotic STHs transmission in sympatric populations of NHPs and humans (Cibot et al., 2015; Frias et al., 2019; Ghai, Chapman, et al., 2014; Ghai, Simons, et al., 2014). However, little is known about urban zoonoses and possible reservoir hosts despite having abundant wildlife in most urban habitats in Africa.

Relevant to this context are the strongylid nematodes (genus Oesophagostomum) commonly referred to as nodular worm that parasitise pigs, ruminants, NHPs and humans. Oesophagostomum bifurcum is considered as the principal nodular worm of humans (Terio et al., 2018) while Oesophagostomum stephanostomum infects great apes including chimpanzees (Cibot et al., 2015) and gorillas (Makouloutou et al., 2014). Although the potential for cross-transmission of Oesophagostomum spp. between humans and NHPs has been disputed (Gruijter et al., 2004; Van Lieshout et al., 2005) a novel Oesophagostomum clade infecting humans and sympatric NHPs populations has been described in Uganda (Cibot et al., 2015; Ghai, Chapman, et al., 2014; Ghai, Simons, et al., 2014). Therefore, localised research to determine the zoonotic risk and the role of NHPs as potential reservoirs, is imperative.

To formulate effective control measures, accurate diagnosis and genetic characterisation of nematodes is vital (Pouillevet et al., 2017). Comparatively, the polymerase chain reaction (PCR) technique is superior to conventional microscopy. Additionally, real-time high-resolution melting (HRM) analysis, a probe-free post-PCR analysis, allows direct characterisation of PCR amplicons (Reed et al., 2007) and has effectively been used to genotype hookworms without the need for sequencing (Ngui et al., 2012). Thus, PCR-HRM is a robust method for answering epidemiological questions that underpin pathogen surveillance and control programs (Villinger et al., 2016).

Previous surveys on helminths have focused on NHPs within wildlife reserves and rural forest habitats (Akinyi et al., 2019; Ghai, Chapman, et al., 2014; Ghai, Simons, et al., 2014; Obanda et al., 2019) leaving information gap on helminth zoonoses originating from free-ranging NHPs within urban and peri-urban centres. In East Africa, the sharing of habitats between NHPs and humans, such as public parks in major cities or watering points in peri-urban towns, may facilitate the cross-species transmission of zoonotic STHs. In Kenya, frequent close contacts between humans and monkeys often occurs in most urban public parks. Resident troops of monkeys often snatch food snacks from visitors, and this act may contaminate salvaged food with nematode infective stages. In addition, visitors often encourage the monkeys to climb on their heads and shoulders for photo shoot, which may represent spread of zoonotic parasites from NHPs to humans via the faecal-oral route. This human and monkey interaction suggests an increased risk for cross-transmission of zoonotic pathogens, and potential epidemiological consequences on pathogen evolution within urban ecology. Thus, determination of the zoonotic risk and the role of NHPs as potential parasite reservoirs in urban centres are imperative. We utilised PCR-HRM and sequencing to investigate the distribution and characterise potentially zoonotic nodular worms in free-ranging
NHPs within densely populated urban and peri-urban centres in Kenya.

\section{2 | MATERIALS AND METHODS}

\section{1 | Study sites}

This study focused on NHPs found in urban centres of Mombasa and Kisumu, and peri-urban centres within Murang'a and Kakamega counties of Kenya (Figure 1). NHPs were captured at six sites: (a) Mombasa $\left(4^{\circ} 03^{\prime} \mathrm{S}, 39^{\circ} 40^{\prime} \mathrm{E}\right)$, a city at the coastal region of Kenya with a population of 1.2 million people. Mombasa has a tropical climate with hot and humid weather, temperature of $29^{\circ} \mathrm{C}$ and $11.2 \mathrm{~mm}$ of precipitation. (b) Kisumu $\left(0^{\circ} 00^{\prime} \mathrm{N}, 34^{\circ} 48^{\prime} \mathrm{E}\right)$, located in western Kenya at the shore of Lake Victoria has a population of about 599,468 people. The lake side town has no true dry season but significant rainfall throughout the year averaging $1321 \mathrm{~mm}$ and temperatures of $24^{\circ} \mathrm{C}$. (c) Murang'a $\left(0^{\circ} 43^{\prime} \mathrm{S}, 37^{\circ} 09^{\prime} \mathrm{E}\right)$ in central Kenya has warm and temperate climate (averaging temperatures of $17.4^{\circ} \mathrm{C}$ and $1,590 \mathrm{~mm}$ of rainfall) and a population of about 1.06 million people. (d) NHPs were sampled from Kakamega County, Western Kenya. This region has a population of about 1.8 million people and temperatures average $20.4^{\circ} \mathrm{C}$ with $1971 \mathrm{~mm}$ of rainfall annually. Here, NHPs were captured from three peri-urban townships namely Buyangu $\left(0^{\circ} 19^{\prime} \mathrm{N} 34^{\circ} 57^{\prime} \mathrm{E}\right)$, Isecheno $\left(0^{\circ} 17^{\prime} \mathrm{N} 34^{\circ} 51^{\prime} \mathrm{E}\right)$ and Malava $\left(0^{\circ} 26^{\prime} \mathrm{N} 43^{\circ} 51^{\prime} \mathrm{E}\right)$.

\subsection{Animal trapping and sample collection}

Animal sampling was opportunistic. NHPs trapped were targeted for translocation to wildlife reserves because they were a public menace in urban areas or were regarded as pests by small-scale farmers within peri-urban areas. They were trapped as previously described (Jeneby et al., 2011) following the guidelines for animal trapping and sampling after appropriate ethical review committee approval was received. Age group and sex of the trapped animals was determined according to Brett et al. (1982). Faecal samples were collected from the rectum via swabbing and stored in $70 \%$ ethanol for molecular assay. Where only one NHP was trapped in a cage, defecated material was collected and stored in $10 \%$ formalin for parasitological assays. If more than one animal was trapped in a cage, the faecal material in the cage was not collected because sample identity could not be confirmed. The samples were transported in a cool box with dry ice $\left(-78.5^{\circ} \mathrm{C}\right)$ to the laboratory .

\section{3 | Non-human primates sampled}

A total of 86 NHPs: 41 African green monkeys (Chlorocebus aethiops), 30 olive baboons (Papio anubis anubis), 5 blue monkeys (Cercopithecus mitis stuhlmanni) and 10 red-tailed monkeys (Cercopithecus ascanius 

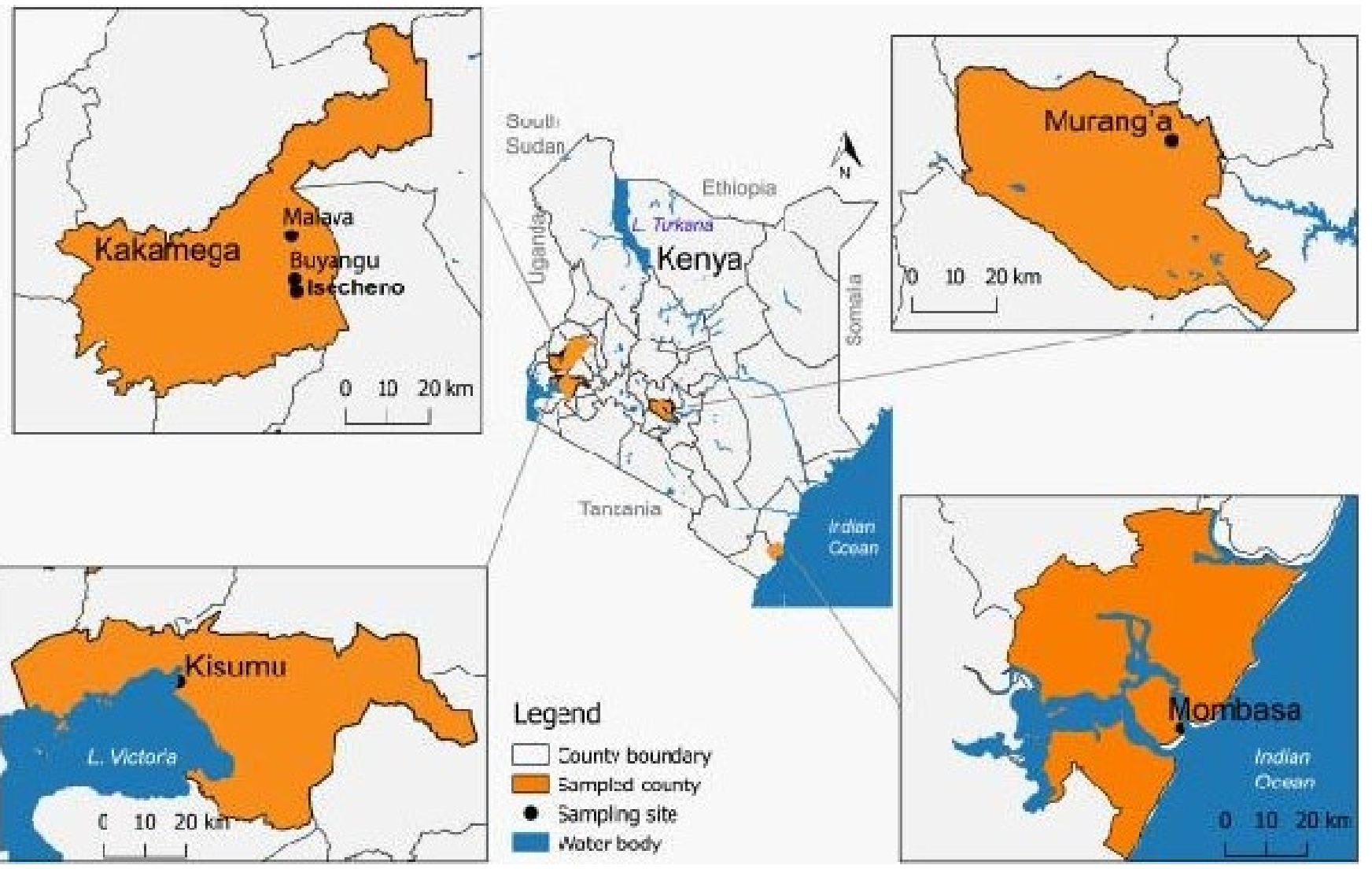

FIGURE 1 Urban and peri-urban sampling centres. A map of Kenya (centre) and the four urban and peri-urban sampling counties (orange shades) namely Kakamega, Murang'a, Kisumu and Mombasa. The black circles show the sampling points in the centres

schmidt's) were trapped and sampled in situ. The species caught from each location,their age category and sex are shown in Table 1. All 86 animals were rectal swabbed for samples while 69 that were individually trapped over the study period had extra faecal samples collected from their cages. In some cases where two or more animals were trapped in a single cage, it was impossible to utilise any of the excreta because of sample identity.

\section{4 | Parasitological examination}

Formal-ether sedimentation and sheathers sugar floatation techniques (Lee et al., 2010) were used for microscopic helminth detection. In both approaches, the slides were examined at 400X in duplicates in a Leica DM2000 LED microscope equipped with a digital camera control unit (Leica DFC 450) and representative images captured. Parasites were identified on the basis of egg colour, shape, internal contents and larvae according to (Kouassi et al., 2015).

\subsection{DNA extraction, PCR assay and sequencing}

A total of 86 , ethanol-preserved, rectal swabbed faecal samples were snap-frozen in liquid nitrogen and ground to fine powder. The powder was homogenised with double distilled water as described previously (Phuphisut et al., 2016). Total DNA was extracted from $200 \mu \mathrm{l}$ of the homogenate using the QIAamp DNA stool Mini kit (Qiagen, Hilden, Germany), according to the manufacturer's instructions and stored at $-20^{\circ} \mathrm{C}$.

Oesophagostomum spp. were detected by PCR amplification of ITS2 gene using NC1 forward (5'-ACG TCT GGT TCA GGG TTG TT-3') and NC2 reverse (5'-TTA GTT TCT TTT CCT CCG CT-3') primer pairs (Ghai, Chapman, et al., 2014; Ghai, Simons, et al., 2014). PCR was performed in a RotorGene Q thermocycler (Qiagen, Hilden, Germany) using 10 $\mu \mathrm{M}$ concentrations of each primer, $4 \mu \mathrm{l}$ of 5X HOT FIREPOL Eva Green HRM Mix (Solis Biodyne, Tartu, Estonia) and $2 \mu$ of the DNA template in a $25 \mu \mathrm{l}$ reaction mix. Thermal cycling conditions were as follows: initial denaturation at $95^{\circ} \mathrm{C}$ for $15 \mathrm{~min}$, followed by 35 cycles of denaturation at $95^{\circ} \mathrm{C}$ for $1 \mathrm{~min}$, annealing at $55^{\circ} \mathrm{C}$ for $45 \mathrm{~s}$, and extension at $72^{\circ} \mathrm{C}$ for $45 \mathrm{~s}$ and a final extension at $72^{\circ} \mathrm{C}$ for $5 \mathrm{~min}$. The PCR products were immediately utilised for high resolution melting (HRM) analysis as described (Villinger et al., 2016). Briefly, the amplicons were denatured at $95^{\circ} \mathrm{C}$ for $1 \mathrm{~min}$, annealed at $40^{\circ} \mathrm{C}$ for $1 \mathrm{~min}$ and equilibrated at $65^{\circ} \mathrm{C}$ for $90 \mathrm{~s}$, and then increasing the temperature in $0.1^{\circ} \mathrm{C}$ increments up to $90^{\circ} \mathrm{C}$ with fluorescence acquisition after $2 \mathrm{~s}$ incremental holding periods. The melting curve profile was then analysed using Rotor-Gene Q series software version 2.1 with fluorescence (melting curve) normalised by selecting the linear region before and after melting transition. Melting temperature ( $\mathrm{Tm}$ ) was interpolated from the normalised data as the temperature at $50 \%$ fluorescence. Distinct HRM 


\begin{tabular}{|c|c|c|c|c|c|c|}
\hline Region & Sex & Age group & AGM & $\begin{array}{l}\text { Olive } \\
\text { baboon }\end{array}$ & $\begin{array}{l}\text { Blue } \\
\text { monkey }\end{array}$ & $\begin{array}{l}\text { Red-tailed } \\
\text { monkey }\end{array}$ \\
\hline \multirow{6}{*}{$\begin{array}{l}\text { Mombasa } \\
30\end{array}$} & Male & Juvenile & 1 & & & \\
\hline & 18 & Sub/Adult & 9 & & & \\
\hline & & Adult & 8 & & & \\
\hline & Female & Juvenile & 2 & & & \\
\hline & 12 & Sub/Adult & 2 & 1 & & \\
\hline & & Adult & 7 & & & \\
\hline \multirow{5}{*}{$\begin{array}{l}\text { Kakamega } \\
28\end{array}$} & Male & Juvenile & & 2 & & 1 \\
\hline & 14 & Sub/Adult & & 3 & 1 & 3 \\
\hline & & Adult & & 2 & 1 & 1 \\
\hline & Female & Sub/Adult & & 2 & & 2 \\
\hline & 14 & Adult & & 6 & 3 & 1 \\
\hline \multirow{5}{*}{$\begin{array}{l}\text { Kisumu } \\
21\end{array}$} & Male & Juvenile & & 1 & & \\
\hline & 10 & Sub/Adult & 2 & 3 & & \\
\hline & & Adult & 1 & 3 & & \\
\hline & Female & Sub/Adult & & 1 & & \\
\hline & 11 & Adult & 2 & 6 & & 2 \\
\hline \multirow{3}{*}{$\begin{array}{l}\text { Murang'a } \\
7\end{array}$} & Male & Juvenile & 1 & & & \\
\hline & 5 & Adult & 4 & & & \\
\hline & $\begin{array}{l}\text { Female } \\
2\end{array}$ & Adult & 2 & & & \\
\hline 86 & & & 41 & 30 & 5 & 10 \\
\hline
\end{tabular}

TABLE 1 The total number of NHP species sampled, distribution of each species according to sampling urban regions according to sex and age group profiles, normalised in the range of $80-90^{\circ} \mathrm{C}$, were visually determined for each reaction after completion of HRM data acquisition. PCR-HRM products were further visualised by $2 \%$ agarose gel electrophoresis stained with ethidium bromide. Gel readings were compared with corresponding PCR-HRM melting peaks for consistency with HRM analysis. Representative positive amplicons from PCR-HRM amplification were purified using the QIAquick Gel Extraction Kit (Qiagen, Hilden, Germany), according to the manufacturer's instructions and sequenced.

\section{6 | Phylogenetic analysis}

Consensus sequences for ITS2 rDNA gene were generated from forward and reverse sequence data using Seqtrace version 0.9.0 (Stucky, 2012) and their identity ascertained by BLAST (Altschul et al., 1990) searches of GenBank (Benson et al., 2005). For species identification, a homology cut-off of $97 \%-100 \%$ identity with a GenBank E-value threshold of 1e-130 was used. Sequences generated from this study and ITS2 ribosomal DNA sequences retrieved from GenBank were used for multiple sequence alignments in MAFFT (Edgar, 2004). Subsequently, evolutionary analyses were performed to determine the relatedness and diversity of the Oesophagostomum spp. and Trichostrongylus spp. identified in this study to other nematode species endemic in East Africa. The evolutionary history was inferred using the maximum likelihood method in MEGA7 (Kumar et al., 2018) with bootstrapping at 1,000 replicates. Phylogenetic trees were rendered in iTOL (Letunic \& Bork, 2019).

\section{3 | RESULTS}

\subsection{Microscopic nematode detection}

Microscopic examination of the 69 faecal samples identified nematode eggs and/or larvae in $78.3 \%$ (54/69) of the samples. These included Strongyloides spp., Ascarid spp., Trichuris spp., Oesophagostomum spp. and Enterobius spp. (Figure 2a). Strongyloides eggs and larvae were observed in $25 / 69$ (36.2\%) of the samples while Trichuris eggs were observed in 54/69 (78.3\%) of the samples. Eggs of Enterobius, Ascarid and Oesophagostomum species were each observed in 5/69 (7.2\%) of the faecal samples (Figure $2 b$ ).

\subsection{Molecular detection of nematodes and sequencing}

PCR-HRM analysis (Figure 3) identified Oesophagostomum spp. in 33/86 (38.4\%) of the NHPs. Of these, 21 and 23 animals were infected with $O$. bifurcum and $\mathrm{O}$. stephanostomum respectively while 11 animals were coinfected with both species (Table 2). BLAST searches of GenBank upon 


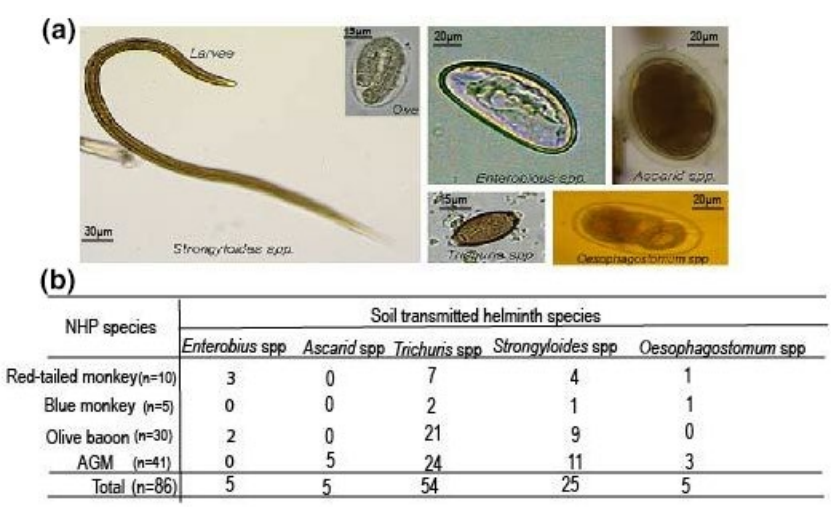

NHPs-Non-human primate species, AGM-Africa green monkey, n-number of individuals sampled per NHP species

FIGURE 2 Nematode infecting NHPs. A, Representative microscopic images of nematode eggs and larvae detected in NHP faecal samples. B, The number of NHP individuals infected with the various nematode species by microscopy

sequencing confirmed their presence and identified another nematode, Trichostrongulus colubriformis. These sequences have been submitted to GenBank under accession numbers (MT184881 to MT184891).

\section{3 | Overall nematode infection by microscopy and PCR-HRM}

Parasitological and molecular assays enabled identification of nematodes in 74/86 (86.05\%) NHP samples. Overall, (35/41) AGMs, $(27 / 30)$ olive baboons, (9/10) red-tailed monkeys and (3/5) blue monkey showed evidence of helminth infection. T. colubriformis and Ascarid spp. were only detected in AGMs, Enterobius in olive baboons and red-tailed monkeys while the rest occurred in all the NHPs (Figure 4). Two AGMs sampled in Mombasa were infected with $T$. colubriformis. Infection varied across the sampling sites with 89.3\% (25/28) in Kakamega, 81.0\% (7/21) in Kisumu, 85.7\% (6/7) in Murang'a and $86.6 \%(26 / 30)$ in Mombasa. Ascarid infections occurred only in animals from Murang'a, T. colubriformis in Mombasa while Enterobius occurred in Kisumu and Kakamega.

\subsection{Helminth co-infections as identified by microscopy and PCR-HRM}

Co-infections were observed across the sampling sites in various NHPs (Figure 5). Trichuris and Strongyloides co-infections were the most common 17.4\% (15/86) and occurred mostly in AGMs from Mombasa 24.4\% (10/41). Strongyloides and Oesophagostomum. co-infection was also detected in $4.7 \%$ (4/86) of all the NHPs except AGMs. Coinfections with three nematodes were observed in all NHPs except the red-tailed monkey and were absent in NHPs trapped from Kisumu and Murang'a. Co-infections with four nematodes were observed in $8.1 \%$ $(7 / 86)$ of the NHPs. Mixed infection with the two Oesophagostomum species occurred in 11 NHPs as shown in Table 2.

\section{5 | Phylogenetic analysis}

The phylogenetic tree obtained resolved into three distinct clades of $O$. stephanostomum, $O$. bifurcum and $T$. (a)

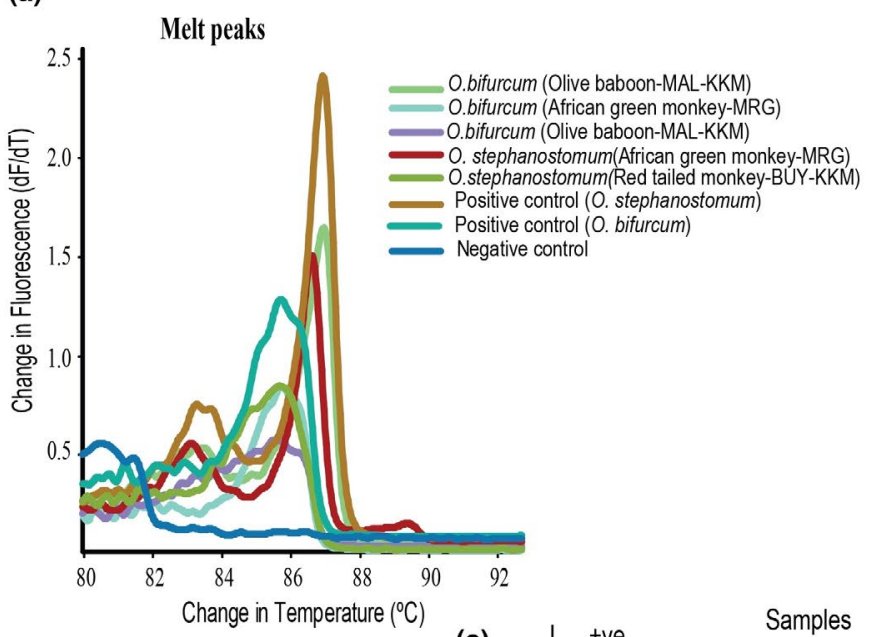

(b)

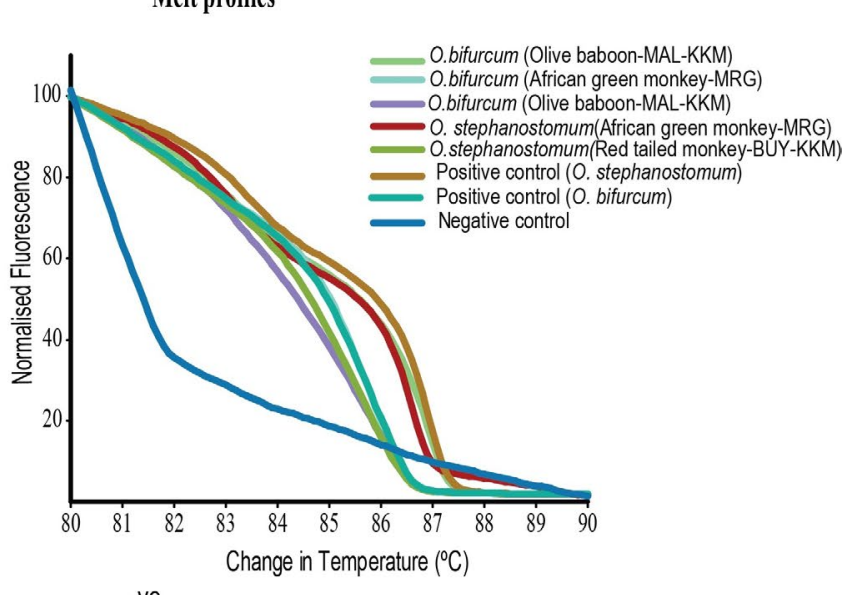

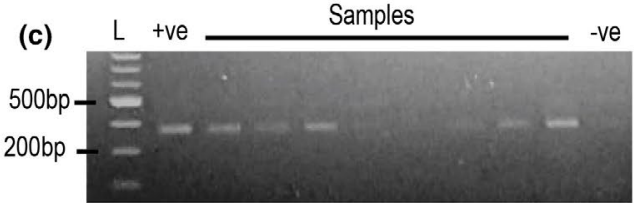

FIGURE 3 Molecular detection of Oesophagostomum spp. by PCR-HRM and gel electrophoresis. A, Representative PCR-HRM melt peaks and B, Melt profiles of O. bifurcum and O. stephanostomum from rectal swabs of sampled NHPs (BUY: Buyangu; MAL: Malava; KKM: Kakamega, and MRG: Murang'a). C, Gel electrophoresis size separation of the amplified products(280bp). L, +ve and -ve represent the DNA ladder, positive and negative controls respectively 


\begin{tabular}{lllll}
\hline Species & $\begin{array}{c}\text { Number } \\
\text { sampled }\end{array}$ & O. stephanostomum & O. bifurcum & $\begin{array}{c}\text { O. stephanostomum } \\
\text { and O. bifurcum }\end{array}$ \\
\hline $\begin{array}{c}\text { Africa green } \\
\text { monkey }\end{array}$ & 41 & $12(29.3 \%)$ & $9(21.95 \%)$ & $5(12.19 \%)$ \\
\hline $\begin{array}{l}\text { Olive baboon } \\
\text { Blue Monkey }\end{array}$ & 30 & $9(30.0 \%)$ & $4(13.33 \%)$ & $4(13.33 \%)$ \\
\hline $\begin{array}{l}\text { Red-tailed } \\
\text { monkey }\end{array}$ & 10 & $1(20.0 \%)$ & $3(60.0 \%)$ & $1(20.0 \%)$ \\
\hline Total & 86 & $1(10.0 \%)$ & $3(30.0 \%)$ & $1(10.0 \%)$ \\
\hline
\end{tabular}

TABLE 2 Oesophagostomum spp. infection of non-human primates (NHPs) from urban and peri-urban centres in Kenya

\section{Distribution of nematodes}

(a) According to NHP species

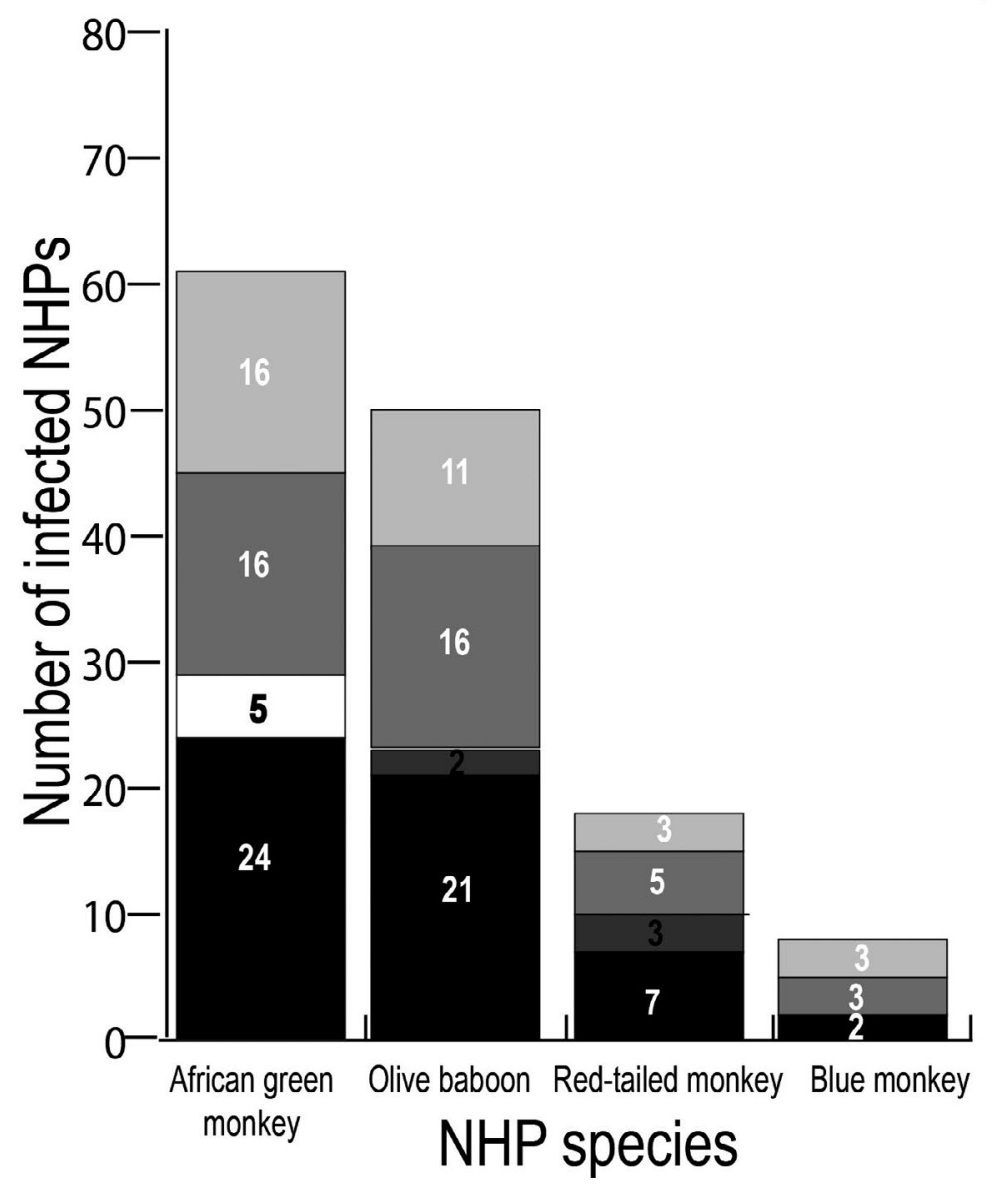

(b) According to sampling region
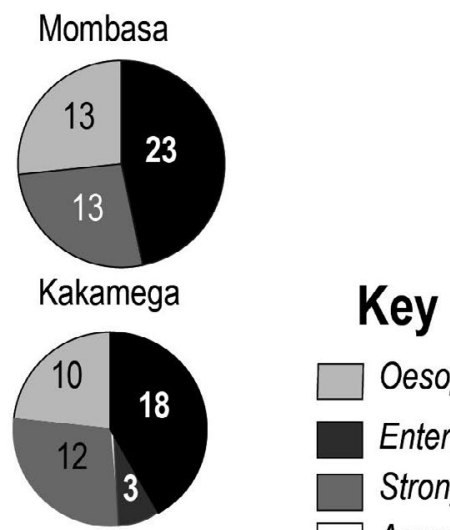

Kisumu

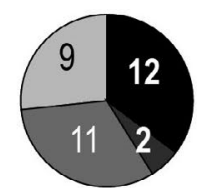

Murang'a

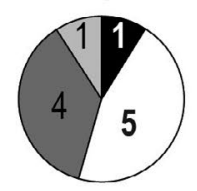

FIGURE 4 Distribution of identified nematode species in the NHPs. The species of nematodes identified in specific NHPs host (graph) and in the four sampling locations (pie charts). The numbers inset show number of infected NHP, with each colour code representing a nematode species

colubriformis (Figure 6). The 0 . stephanostomum clade lacked species or geographical sub-structuring; red-tailed monkeys' sequences and a blue monkey sequence formed one sub-cluster while sequences from AGM and red-tailed monkey formed a second sub-cluster. Contrastingly, the $O$. bifurcum clade grouped the baboon and AGM isolates in different sub-clusters. The cryptic Oesophagostomum species described from Uganda (Ghai, Chapman, et al., 2014; Ghai, Simons, et al., 2014) was phylogenetically distinct from both $O$. stephanostomum and $O$. bifurcum identified in this study. The T. colubriformis clade grouped AGM-derived isolates in a distinct cluster from previous sequence of $T$. colubriformis DNA isolated from yellow baboon in Kenya and those reported from humans in Laos. 
(a) Co-infection with two nematodes

(a) According to NHP species

(b) According to sampling region

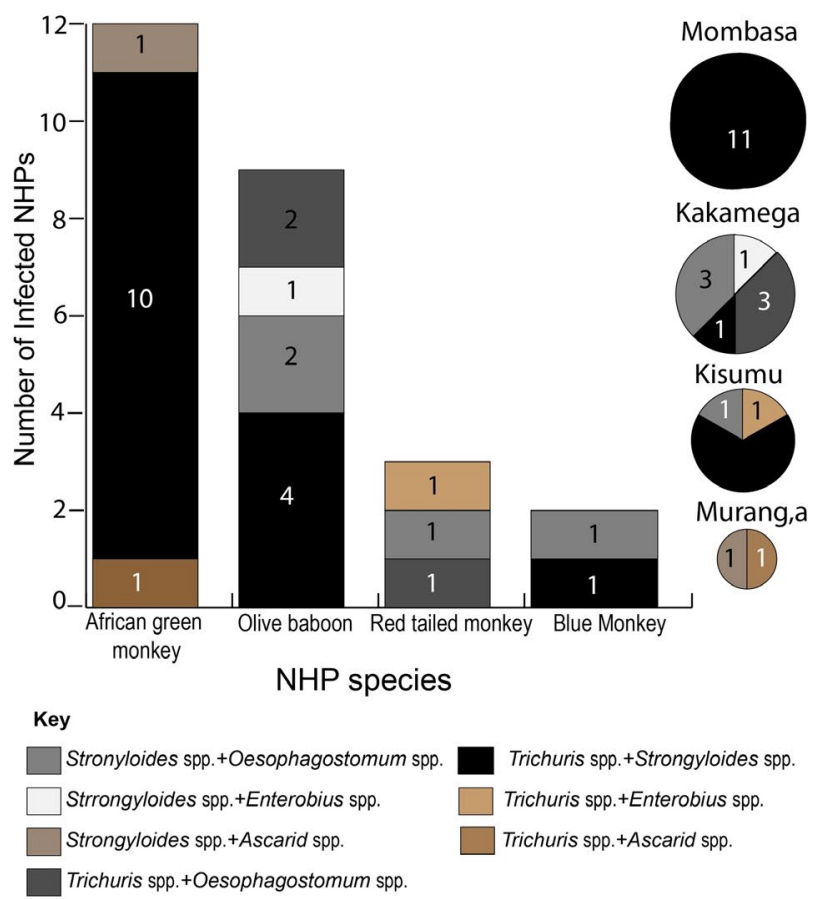

(b) Co-infection with three and four nematodes

(a) According to NHP species

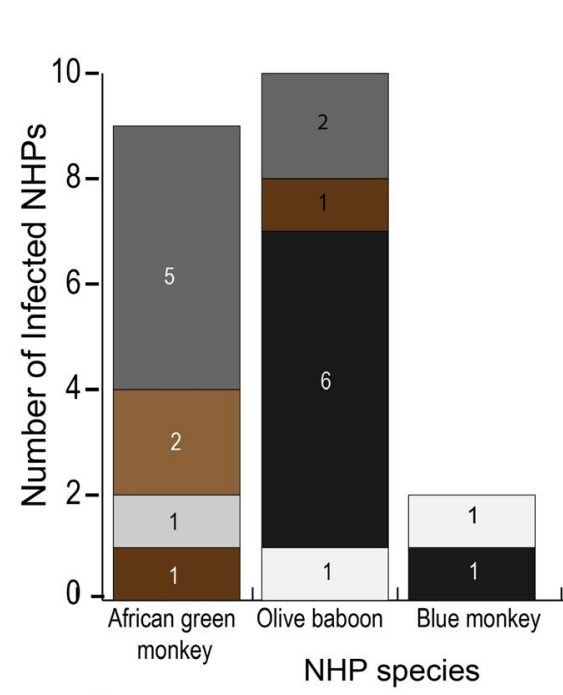

Key

$\square$ Trichuris spp.+Strongyloides spp.+Trichostrongylus spp.+Oesophagostomum spp. Ascarid spp.+Oesophagostomum spp.+Strongyloides spp.

Trichostrongylus spp.+trichuris spp. +oesophagostomum spp. Enterobius spp.+Oesophagostomum spp.+Trichuris spp.

Strongyloides spp.+Oesophagostomum spp.+Trichuris spp. Ascarid spp.+Trichuris spp.+Oesophagostomum spp.

FIGURE 5 Nematodes co-infections in NHPs. A, Co-infections with two nematodes species (column graph) in the various sampling regions (pie chart). B, Co-infections with three and four nematode species (column graph) in the different sampling regions (pie chart). The number of individual NHPs infected is shown inset with each colour code representing a nematode species

\section{4 | DISCUSSION}

The diversity of STHs identified in selected Kenyan urban centres highlights the possible reservoir role played by NHPs in helminths urban ecology. The diversity of nematodes infecting different species of NHPs within urban and peri-urban centres compliments previous molecular surveys that detected helminths in free-ranging NHPs within remote wildlife habitats in Kenya (Akinyi et al., 2019; Obanda et al., 2019). In STHs endemic countries, re-infection has been observed in half of the children treated for intestinal worms (Jia et al., 2012) a phenomenon attributed to re-infection due to persistence of infective worm stages in the environment. As the Kenyan government commits to strengthen helminth control (Mwandawiro et al., 2019) it is faced with the challenge of identifying reservoir hosts. This study provides useful information on possible reservoir hosts of STHs that may contribute to environmental sustenance of zoonotic nematodes in urban and peri-urban regions of Kenya. It also illustrates the possibility of utilising PCR-HRM analysis to efficiently differentiate between Oesophagostomum spp. without the need for sequencing as earlier described for hookworms (Ngui et al., 2012). In this study, PCR-HRM differentiated O. stephanostomum and O. bifurcum co-infecting NHPs (Figure 3 ) as confirmed by sequencing indicating its utility as a non-subjective approach to supplement sequencing for accurate characterisation of nodular worms.
Phylogenetic reconstruction of nodular worm isolates demonstrated separation of $O$. stephanostomum sequences into a sub-cluster consisting of isolates from red-tailed monkeys and blue monkeys in Kakamega and a sub-cluster consisting of sequences from AGMs sampled in Kisumu and Murang'a indicating that O. stephanostomum is neither geographical nor host species based. In addition, clustering of $\mathrm{O}$. stephanostomum detected in this study with an isolate from human (accession number KR149647.1) indicates close evolutionary relatedness and therefore suggests the potential for this parasite to infect both humans and NHPs. This finding concurs with Cibot et al. (2015) reports of human and NHP infection with O. stephanostomum, suggesting increased risk of transmission for this helminth between primate species. $O$. bifurcum sequences from olive baboons in this study formed a monophyletic cluster with $O$. bifircum sequences from other NHP species retrieved from Genbank. However, the $O$. bifurcum sequence from a chimpanzee sampled in Uganda clustered with sequences recovered from an AGM in Kenya. The two were evolutionarily divergent from the rest of the sequences within this cluster. This points to potentially new host species since O.bifurcum has not been previously reported in AGMs in Kenya and is not commonly described in chimpanzees. Because transmission occurs via the ingestion of the infective third-stage larvae present in contaminated food or water, oesophagostomosis is a potential zoonotic risk when infected NHPs and humans share the same habitats. 


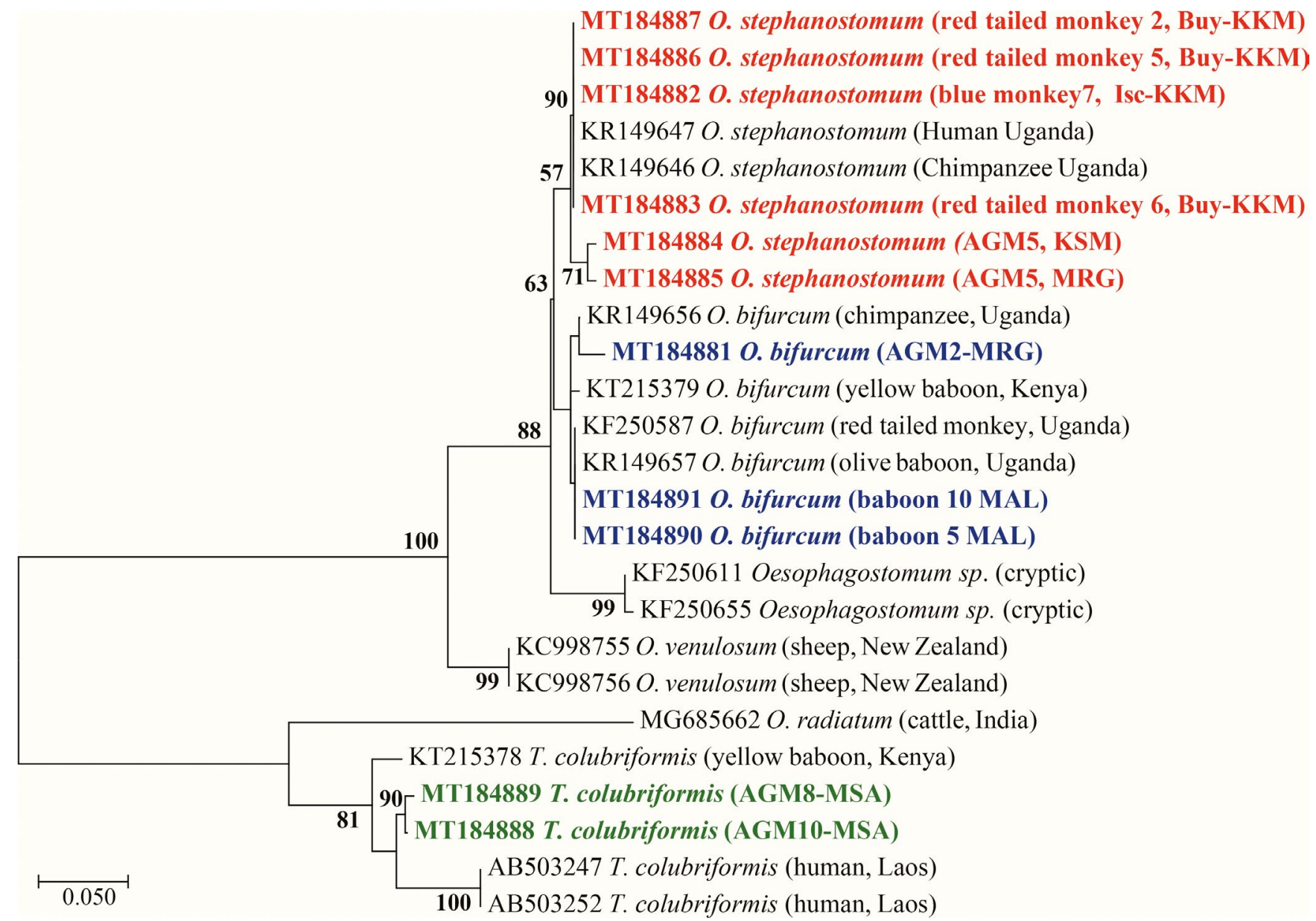

FIGURE 6 Maximum likelihood tree based on the ITS2 rDNA gene. Sequences generated in this study are shown in bold and correspond to the NHP species, its number and the sampling site (Buy: Buyangu; MAL: Malava; Isc: Isecheno; KKM: Kakamega, KSM: Kisumu; MSA: Mombasa; and MRG: Murang'a). O. stephanostomum sequences generated in this study are highlighted in red while O. bifurcum and T. colubriformis sequences are highlighted in blue and green respectively. Bootstrap values are shown as percentages, only values greater than 50 are indicated. Scale bar indicates nucleotide substitutions per site. Evolutionary analyses were conducted in MEGA7

Therefore, intervention strategies to combat oesophagostomosis need to factor NHPs as potential reservoirs. Sequencing data also confirmed infection by T. colubriformis in olive baboons. T. colubriformis sequences generated in this study were distinct from the Trichostrongylus spp. isolates from yellow baboon in Kenya (Obanda et al., 2019)which may be as a result of sub structuring according to host species.

Other nematodes identified in this study included Trichuris., Enterobius., Ascarid., Strongyloides. and Trichostrongylus species. Trichuris trichiura, has been detected in different primate species in Kenya (Akinyi et al., 2019; Mbora \& McPeek, 2009) and experimentally transmitted from NHPs to humans (Monteiro et al., 2007) providing evidence of its zoonotic potential. In addition, a single taxon was found to be both human and NHPs infective in a Trichuris host diversity study (Ghai, Chapman, et al., 2014; Ghai, Simons, et al., 2014). NHPs are known to be the major hosts of $S$. stercoralis and, especially, S. fuelleborni. A peculiarity of Strongyloides spp., is their ability to penetrate the host's skin and/or autoinfect making them burdensome helminths causing long-term suffering. Identification of Strongyloides in this study is thus of public health concern. Klaus et al. (2017) demonstrated Ascarid spp. cross-infection and zoonotic potential between human and NHPs. Nematodes in the genus Trichostrongylus are known to infect humans, wild animals and herbivorous animals (Obanda et al., 2019). Primates including humans become infected due to environmental contamination. Where NHPs, ruminants and humans live in sympatry, T. colubriformis is a perpetual public health burden thus its presence poses a potential risk of transmission in the study areas. Contrary to previous studies (Munene et al., 1998; Obanda et al., 2019) AGMs in this study were infected with $O$. bifurcum which adds AGMs as reservoir host species to the epidemiology of nodular worm. Overall, a complex anthropo-zoonotic transmission cycle may be maintained in the study regions.

Consistent with other studies (Akinyi et al., 2019; Klaus et al., 2017), we also observed co-infection with multiple nematode species. Parasites co-occurring within a single host interact in a variety of ways that influence their abundance, distribution and the dynamics of one another (Pedersen \& Fenton, 2007). One such interaction is the immune modulation. A helminth induced immunosuppression caused by infection with one helminth species 
may strongly alter a host's response to subsequent infection by other species enhancing the likelihood of co-infection (Cox, 2001). Furthermore, mechanisms related to host exploitation can be modified to ultimately enhance coexistence. For instance, the distinct spatial niches of worms within a host, for example, Ascaris lumbricoides predilection in jejunum while Trichuris trichiura resides in the cecum reduces levels of resources competition between the two worms. Predominance of Trichuris and Strongyloides co-infections in this study may be due to their abundance in the faecal samples as they were the most dominant infections. Given the ubiquity of co-infection in nature, and the effects co-infecting parasites are likely to have on one another, interactions among parasites may be a major force generating variation in the transmission of disease and in shaping infectious disease dynamics. While most studies on intestinal helminths of free-ranging NHP rely on faecal samples collected after defecation in the wild, challenges in interpretation of results may arise due to collection of several samples from the same animal (Gillespie et al., 2010). In the current study, stool samples could be traced back to the individual animal having collected the sample from the rectum of the animals or freshly excreted faecal sample from trapping cages with single animal.

The number of nematode infections in NHPs was highest in Mombasa and Kakamega while Murang'a had the least cases of infections. The difference in infection among these locations was statistically significant $\left(\chi^{2}=5.991, d f=2, p<0.0001\right.$ ) By comparison, the prevalence of all STHs in human population in Kenya is highest in coastal and western regions (Brooker \& Michael, 2000; Masaku et al., 2017). One explanation for high cases of infection in Mombasa could be a coastal habitat providing ideal environmental conditions such as humidity and warmth for egg development. Although Kisumu is also warm, humidity is much lower. Additionally, frequent recurrence of STHs at the same location may facilitate environmental accumulation of infective parasite stages and could result in reinfection. This is especially relevant in the case of reservoir hosts, since adult worms' lifespan is typically longer than annual periods when environmental conditions favours transmission thus maintaining overall endemicity.

A key limitation of this study is that we were unable to sample humans within the studied regions. Our sampling was opportunistic because we focused on NHPs that had been targeted for translocation to national reserves. In most cases, surveillance of zoonotic pathogens under the 'One Health' approach recommends that humans, wildlife and domestic animals that share same habitats are screened concurrently. Our approach to phylogenetic analysis addressed this limitation by comparing our generated sequences to those of nematodes isolated from humans and domestic animal faecal material. Although our data provide baseline information on the potential zoonotic risk of gastrointestinal nematodes in urban centres, we recommend further surveillance of the nematodes using the one health approach. Since NHPs serve as sentinels for surveillance of emerging diseases, the rich taxonomic diversity of nematodes detected NHPs from selected urban centres in Kenya could be an important reference to the helminth prevalence in altered urban habitats. In addition, the detection of Oesophagostomum species in free-ranging NHPs within densely populated urban centres is of public health interest because of the zoonotic nature of these nematodes, especially $O$. bifurcum, a parasite that can be lethal to humans.

\section{5 | ETHICAL NOTE}

Animal trapping, sedation and sampling were undertaken with approval from and according to the guidelines of Institute of Primate Research (IPR) Institutional Scientific Review Committee and the Department of Veterinary and Capture Services of the Kenya Wildlife Service (KWS), Nairobi (Review number, ISERC/04/18).

\section{ACKNOWLEDGEMENTS}

The authors gratefully acknowledge the institutional support of the Institute of Primate Research (IPR), Kenya and the Kenya Wildlife Service (KWS). We also thank Dr. Moses Otiende and Mr. Samson Mutura for their assistance with the molecular experiments. This study received financial support from the Consortium for National Health Research (CNHR) grant, Kenya (Project number RCDG041/2012) and by a Global Enhancement Fund grant, USA (2017) held by Prof. Susan Alberts, Duke University.

\section{CONFLICT OF INTEREST}

The authors declare that they have no conflict of interest.

\section{AUTHORS CONTRIBUTION}

MJ, VO, PM and MA formulated the project. MJ, MA, IL, PM and GE conducted fieldwork. PM, MJ and MA conducted laboratory analysis. MJ, EM, VO, RN and PM analysed the data. MJ, MA, PM, VO and EM drafted the manuscript. All the authors revised the manuscript and approved the final manuscript draft.

\section{PEER REVIEW}

The peer review history for this article is available at https://publo ns.com/publon/10.1002/vms3.424.

\section{ORCID}

Peris Mbuthia (iD https://orcid.org/0000-0002-8568-512X

Richard Nyamota iD https://orcid.org/0000-0002-9569-1953

Maamun Jeneby iD https://orcid.org/0000-0002-3001-402X

\section{REFERENCES}

Akinyi, M. Y., Jansen, D., Habig, B., Gesquiere, L. R., Alberts, S. C., \& Archie, E. A. (2019). Costs and drivers of helminth parasite infection in wild female baboons. Journal of Animal Ecology, 88(7), 1029-1043. https://doi.org/10.1111/1365-2656.12994

Altschul, S. F., Gish, W., Miller, W., Myers, E. W., \& Lipman, D. J. (1990). BLAST_article.pdf. Journal of Molecular Biology, 215(3), 403-410. https://doi.org/10.1016/S0022-2836(05)80360-2

Benson, D. A., Karsch-Mizrachi, I., Lipman, D. J., Ostell, J., \& Wheeler, D. L. (2005). GenBank. Nucleic Acids Research, 33(Database issue), 34-38. https://doi.org/10.1093/nar/gki063 
Brett, F. L., Turner, T. R., Jolly, C. J., \& Cauble, R. G. (1982). Trapping baboons and vervet monkeys from wild, free-ranging populations. The Journal of Wildlife Management, 46(1), 164-174. https://doi. org/10.2307/3808419

Brooker, S., \& Michael, E. (2000). The potential of geographical information systems and remote sensing in the epidemiology and control of human helminth infections. Advances in Parasitology, 47, 245-288. https://doi.org/10.1016/s0065-308x(00)47011-9

Cibot, M., Guillot, J., Lafosse, S., Bon, C., Seguya, A., \& Krief, S. (2015). Nodular worm infections in wild non-human primates and humans living in the Sebitoli area (Kibale National Park, Uganda): Do high spatial proximity favor zoonotic transmission? PLoS Neglected Tropical Diseases, 9(10), 1-17. https://doi.org/10.1371/journal.pntd.0004133

Cox, F. E. G. (2001). Concomitant infections, parasites and immune responses. Parasitology, 122(S1), S23-S38. https://doi.org/10.1017/ s003118200001698x

DeGruijter, J. M., Ziem, J., Verweij, J. J., Polderman, A. M., \& Gasser, R. B. (2004). Genetic substructuring within Oesophagostomum bifurcum (Nematoda) from human and non-human primates from Ghana based on random amplified polymorphic DNA analysis. The American Journal of Tropical Medicine and Hygiene, 71(2), 227-233. https:// doi:10.4269/ajtmh.2004.71.227

Eastwood, G., Sang, R. C., Guerbois, M., Taracha, E. L., \& Weaver, S. C. (2017). Enzootic circulation of Chikungunya virus in East Africa: Serological evidence in non-human Kenyan primates. The American Journal of Tropical Medicine and Hygiene, 97(5), 1399-1404. https:// doi.org/10.4269/ajtmh.17-0126

Edgar, R. C. (2004). MUSCLE: multiple sequence alignment with high accuracy and high throughput. Nucleic Acids Research, 32(5), 17921797. https://doi.org/10.1093/nar/gkh340

Frias, L., Stark, D. J., Salgado Lynn, M., Nathan, S., Goossens, B., Okamoto, M., \& Maclntosh, A. J. J. (2019). Molecular characterization of nodule worm in a community of Bornean primates. Ecology and Evolution, 9(7), 3937-3945. https://doi.org/10.1002/ece3.5022

Ghai, R. R., Chapman, C. A., Omeja, P. A., Davies, T. J., \& Goldberg, T. L. (2014). Nodule worm infection in humans and wild primates in Uganda: Cryptic species in a newly identified region of human transmission. PLoS Neglected Tropical Diseases, 8(1), 39. https://doi. org/10.1371/journal.pntd.0002641

Ghai, R. R., Simons, N. D., Chapman, C. A., Omeja, P. A., Davies, T. J., Ting, N., \& Goldberg, T. L. (2014). Hidden population structure and cross-species transmission of whipworms (Trichuris sp.) in humans and non-human primates in Uganda. PLoS Neglected Tropical Diseases, 8(10), e3256. https://doi.org/10.1371/journal.pntd.0003256

Gillespie, T. R., Lonsdorf, E. V., Canfield, E. P., Meyer, D. J., Nadler, Y., Raphael, J., Pusey, A. E., Pond, J., Pauley, J., Mlengeya, T., \& Travis, D. A. (2010). Demographic and ecological effects on patterns of parasitism in eastern chimpanzees (Pan troglodytes schweinfurthii) in Gombe National Park, Tanzania. American Journal of Physical Anthropology, 143(4), 534-544. https://doi.org/10.1002/ajpa.21348

Jeneby, M. M., Suleman, M. A., Akinyi, M., Ozwara, H., \& Kariuki, T. (2011). Prevalence of Babesia microti in free-ranging baboons and African green monkeys, Journal of Parasitology, 97(1), 63-67. https:// doi.org/10.1645/GE-2391.1

Jia, T. W., Melville, S., Utzinger, J., King, C. H., \& Zhou, X. N. (2012). Soiltransmitted helminth reinfection after drug treatment: A systematic review and meta-analysis. PLoS Neglected Tropical Diseases, 6(5), https://doi.org/10.1371/journal.pntd.0001621

Klaus, A., Zimmermann, E., Röper, K. M., Radespiel, U., Nathan, S., Goossens, B., \& Strube, C. (2017). Co-infection patterns of intestinal parasites in arboreal primates (proboscis monkeys, Nasalis larvatus) in Borneo. International Journal for Parasitology: Parasites and Wildlife, 6(3), 320-329. https://doi.org/10.1016/j.ijppaw.2017.09.005

Kooriyama, T., Hasegawa, H., Shimozuru, M., Tsubota, T., Nishida, T., \& Iwaki, T. (2012). Parasitology of five primates in Mahale Mountains
National Park, Tanzania. Primates, 53(4), 365-375. https://doi. org/10.1007/s10329-012-0311-9

Kouassi, R. Y. W., McGraw, S. W., Yao, P. K., Abou-Bacar, A., Brunet, J., Pesson, B., Bonfoh, B., N'Goran, E. K., \& Candolfi, E. (2015). Diversity and prevalence of gastrointestinal parasites in seven non-human primates of the Taï National Park, Côte d'Ivoire. Parasite, 22, https://doi org/10.1051/parasite/2015001

Kumar, S., Stecher, G., Li, M., Knyaz, C., \& Tamura, K. (2018). MEGA X: Molecular evolutionary genetics analysis across computing platforms. Molecular Biology and Evolution, 35(6), 1547-1549. https://doi. org $/ 10.1093 / \mathrm{molbev} / \mathrm{msy} 096$

Lee, J., Kang, S., Kim, N., Lee, C., Ahn, K., Kwon, H., Park, C., \& Kim, S. (2010). Investigation of helminths and protozoans infecting old world monkeys: Captive vervet, cynomolgus, and rhesus monkeys. 'Korean Journal of Veterinary Research, 50 (4), 273-277.

Letunic, I., \& Bork, P. (2019). Interactive tree of life (iTOL) v4: Recent updates and new developments. Nucleic Acids Research, 47(W1), W256-W259. https://doi.org/10.1093/nar/gkz239

Makouloutou, P., Mbehang Nguema, P. P., Fujita, S., Takenoshita, Y., Hasegawa, H., Yanagida, T., \& Sato, H. (2014). Prevalence and genetic diversity of Oesophagostomum stephanostomum in wild lowland gorillas at Moukalaba-Doudou National Park, Gabon. Helminthologia (Poland), 51(2), 83-93. https://doi.org/10.2478/s11687-014-0214-y

Masaku, J., Mutungi, F., Gichuki, P. M., Okoyo, C., Njomo, D. W., \& Njenga, S. M. (2017). High prevalence of helminths infection and associated risk factors among adults living in a rural setting, central Kenya: A cross-sectional study. Trophical Medicine and Health, 45(1), https:// doi.org/10.1186/s41182-017-0055-8

Mbora, D. N. M., \& McPeek, M. A. (2009). Host density and human activities mediate increased parasite prevalence and richness in primates threatened byhabitatloss and fragmentation. Journal of Animal Ecology, 78(1), 210-218. https://doi.org/10.1111/j.1365-2656.2008.01481.x

Monteiro, R. V., Dietz, J. M., Beck, B. B., Baker, A. J., Martins, A., \& Jansen, A. M. (2007). Prevalence and intensity of intestinal helminths found in free-ranging golden lion tamarins (Leontopithecus rosalia, Primates, Callitrichidae) from Brazilian Atlantic forest. Veterinary Parasitology, 145(1-2), 77-85. https://doi.org/10.1016/j.vetpar.2006.12.004

Munene, E., Otsyula, M., Mbaabu, D. A. N., Mutahi, W. T., Muriuki, S. M. K., \& Muchemi, G. M. (1998). Helminth and protozoan gastrointestinal tract parasites in captive and wild-trapped African non-human primates. Veterinary Parasitology, 78(3), 195-201. https://doi. org/10.1016/S0304-4017(98)00143-5

Mwandawiro, C., Okoyo, C., Kihara, J., Simiyu, E., Kepha, S., Campbell, S. J., Freeman, M. C., Brooker, S. J., \& Njenga, S. M. (2019). Results of a national school-based deworming programme on soil-transmitted helminths infections and schistosomiasis in Kenya: 2012-2017. Parasites and Vectors, 12(1), 1-18. https://doi.org/10.1186/s1307 1-019-3322-1

Ngui, R., Lim, Y. A. L., \& Chua, K. H. (2012). Rapid Detection and identification of human hookworm infections through high resolution melting (HRM) analysis. PLoS One, 7(7), https://doi.org/10.1371/journ al.pone.0041996

Obanda, V., Maingi, N., Muchemi, G., Ng'ang'a, C. J., Angelone, S., \& Archie, E. A. (2019). Infection dynamics of gastrointestinal helminths in sympatric non-human primates, livestock and wild ruminants in Kenya. PLoS One, 14(6), 1-19. https://doi.org/10.1371/journal.pone.0217929

Pedersen, A. B., \& Fenton, A. (2007). Emphasizing the ecology in parasite community ecology. Trends in Ecology and Evolution, 22(3), 133-139. https://doi.org/10.1016/j.tree.2006.11.005

Phuphisut, O., Maipanich, W., Pubampen, S., Yindee, M., Kosoltanapiwat, N., Nuamtanong, S., Ponlawat, A., \& Adisakwattana, P. (2016). Molecular identification of the strongyloid nematode Oesophagostomum aculeatum in the Asian wild elephant Elephas maximus. Journal of Helminthology, 90(4), 434-440. https://doi. org/10.1017/S0022149X15000541 
Pouillevet, H., Dibakou, S. E., Ngoubangoye, B., Poirotte, C., \& Charpentier, M. J. E. (2017). A comparative study of four methods for the detection of nematode eggs and large protozoan cysts in mandrill faecal material. Folia Primatologica, 88(4), 344-357. https://doi. org/10.1159/000480233

Reed, G. H., Kent, J. O., \& Wittwer, C. T. (2007). High-resolution DNA melting analysis for simple and efficient molecular diagnostics. Pharmacogenomics, 8, 597-608. https://doi.org/10.2217/14622 416.8.6.597

Stucky, B. J. (2012). Seqtrace: A graphical tool for rapidly processing DNA sequencing chromatograms. Journal of Biomolecular Techniques, 23(3), 90-93. https://doi.org/10.7171/jbt.12-2303-004

Terio, K. A., Lonsdorf, E. V., Kinsel, M. J., Raphael, J., Lipende, I., Collins, A., Li, Y., Hahn, B. H., Travis, D. A., \& Gillespie, T. R. (2018). Oesophagostomiasis in non-human primates of Gombe National Park. Tanzania. American Journal of Primatology, 80(1), 1-9. https:// doi.org/10.1002/ajp.22572

Van Lieshout, L., De Gruijter, J. M., Adu-Nsiah, M., Haizel, M., Verweij, J. J., Brienen,E.A.T.,Gasser,R.B.,\&Polderman,A.M.(2005).Oesophagostomum bifurcum in non-human primates is not a potential reservoir for human infection in Ghana. Tropical Medicine and International Health, 10(12), 13151320. https://doi.org/10.1111/j.1365-3156.2005.01527.x

Villinger, J., Mbaya, M. K., Ouso, D., \& Kipanga, P. N. (2016). Arbovirus and insect-specific virus discovery in Kenya by novel six genera multiplex high-resolution melting analysis. Molecular Ecology Resources, 17(3), 466-480. https://doi.org/10.1111/1755-0998.12584

How to cite this article: Mbuthia P, Murungi E, Owino V, et al. Potentially zoonotic gastrointestinal nematodes co-infecting free ranging non-human primates in Kenyan urban centres. Vet Med Sci. 2021;7:1023-1033. https://doi.org/10.1002/ vms3.424 